InnOvaciOnes de NegOciOs 16(32): 166-198

(C) 2019 UANL, Impreso en México (ISSN: 2007-1191)

Recepción: 23 Mayo de 2019 Aceptación: 22 Junio de 2019

\title{
Medición de tiempo y frecuencia en las empresas de telecomunicaciones y sus implicaciones económicas (Measurement of time and frequency in telecommunication companies and their economic implications)
}

\author{
Oscar Alonso Ordaz Pérez, Luz María Marín Aboytes, Denise \\ Gómez Hernández \\ Universidad Autónoma de Querétaro \\ alonso.ordaz@gmail.com,Imarin709@gmail.com, denise.gomez@uaq.mx
}

\begin{abstract}
The objective of this paper is to determine the impact on GDP of the traffic of information measured in time and frequency of the telephone networks in telecommunications companies. In this way, a quantitative approach was carried out using different econometric studies, based on the information obtained from the Federal Institute of Telecommunications. In order to study the behavior of telecommunications in the national GDP and the evolution of the information transfer of telephone networks. The results show that telecommunications have contributed more and more in the GDP, the contribution varies based on the observed quarter. It also identifies that the telephone networks are the ones that make the greatest investment in the infrastructure destined in the telecommunications sector. With regard to information traffic, it is observed that in recent months there has been a decrease in the information traffic of telephone calls.
\end{abstract}

Key words: Gross Domestic Product, Telephone Networks, Information traffic, Econometrics.

Medición de tiempo y frecuencia en las empresas 
JEL: C02, C53.

Resumen: El objetivo del presente trabajo es determinar el impacto en el PIB del tráfico de información, medido en tiempo y frecuencia de las distintas redes de telefonía en las empresas de telecomunicaciones. Para ello, se utilizó un enfoque cuantitativo utilizando diferentes estudios econométricos, con base en información obtenida del Instituto Federal de Telecomunicaciones. Los resultados muestran que las telecomunicaciones han aportado cada vez más en el PIB de México y la contribución varía dependiendo del trimestre observado. También se identifica que las redes de telefonía son las que realizan mayor inversión en la infraestructura destinada en el sector de telecomunicaciones. Con respecto al tráfico de información se observa que en los últimos meses existe una disminución del tráfico de información de las llamadas telefónicas.

Palabras Clave: Producto Interno Bruto, Redes de Telefonía, Tráfico de información, econometría

\section{Introducción}

La relación del Producto Interno Bruto (PIB) y las telecomunicaciones se ha estudiado con anterioridad, comprobando que las telecomunicaciones son muy importantes en el desarrollo de los países (García, 2007). Piedras (2012) menciona que la contribución de las telecomunicaciones en el PIB de México se encuentra en un $3.5 \%$, es por esto que es

Ordaz, O., Marín, L., \& Gómez, D. 
importante identificar como ha sido la inversión en infraestructura en los últimos años de las empresas de telecomunicaciones y cuál ha sido su uso, el cual se estudia mediante el análisis del tráfico de información con su respectiva medida de tiempo (Erlang).

El objetivo del presente trabajo es determinar el impacto en el PIB del tráfico de información medido en tiempo y frecuencia de las distintas redes de telefonía en las empresas de telecomunicaciones. Para ello, se realiza un análisis econométrico basado en la prueba estadística Chow para determinar el comportamiento de las variables PIB de México, PIB de telecomunicaciones, Inversión en infraestructura, y el tráfico de información de las llamadas telefónicas. La investigación se llevó a cabo en distintas facetas:

- Análisis de la contribución de las telecomunicaciones al PIB nacional.

- Análisis de la inversión en infraestructura de las diferentes empresas de telecomunicaciones.

- Estudio del comportamiento del tráfico de información en las llamadas telefónicas.

Medición de tiempo y frecuencia en las empresas 
El estudio se realizó utilizando los datos del año 2000 al 2016 para las variables del PIB de México y el PIB de las telecomunicaciones, esto para medir la relación entre el PIB de México y el PIB de las telecomunicaciones. La siguiente base de datos que se tomó en cuenta es del año 2013 al 2016, esto para observar cual ha sido el comportamiento de la inversión en infraestructura de las distintas compañías de telecomunicaciones. La última base se toma del periodo de octubre de 2015 a junio de 2017, esto para analizar cómo ha sido el tráfico de información, con base en la inversión generada.

\section{Marco teórico}

\section{Producto interno bruto}

El Producto Interno Bruto (PIB) nace ante la necesidad de medir el crecimiento económico de una nación (Jones, 2009). Kuznetz (1934) fue el pionero del cálculo del PIB determinando diferentes variables y métodos estadísticos. Jones (2009), Dornbusch, Fisher y Startz. (2004) define el PIB como "el valor del mercado de los bienes y servicios finales producidos en una economía durante un determinado periodo". (Jones, 2009, Dornbusch et al., 2004).

Ordaz, O., Marín, L., \& Gómez, D. 
A pesar que Kuznetz (1934) realiza diferentes estudios del cálculo del PIB, llega a la conclusión que es difícil medir el crecimiento económico ya que existen variables que son difíciles de medir tal y como lo es la calidad del crecimiento, los costes y beneficios y los periodos de corto y largo plazo. Otros autores como Stiglitz, Sen y Firoussi (2008) están de acuerdo con Simon Kusnetz que el PIB no puede medir adecuadamente el crecimiento de un país, principalmente por el proceso de medición y la forma de calcular los resultados.

Ante esto se pueden mencionar otros índices que se pueden utilizar para el cálculo del crecimiento económico tal y como lo son: El Indicador de Progreso Genuino o Real (IPG), PIB verde, Índice de Desarrollo Humano (IDH), Felicidad Interior Bruta (FIB), Índice de Progreso Social, Índice de Capacidades Básicas, Sistema de Contabilidad Ambiental Económica Integrada (SCAEI), Indicadores tipo huellas; la huella ecológica y la huella hídrica (Galindo, 2015).

Aunque se menciona que el PIB no es el mejor indicador y que existen otros índices para el cálculo del crecimiento económico Mankiw (2006) y Jones (2009) consideran que el PIB aún es el mejor indicador para obtener los mejores resultados de una economía, ya que actualmente existen diferentes

Medición de tiempo y frecuencia en las empresas 
institutos estadísticos que calculan los datos de forma sistemática, con el objetivo de obtener una sola cifra del valor monetario en un determinado momento.

Bajo la premisa de Jones (2009) y Mankiw (2006) que mencionan anteriormente se puede determinar el enfoque vía gasto para medir el PIB, el cual divide los bienes y servicios que se compran en varias categorías (Mankiw, 2006, Jones, 2009; Dornbusch et al., 2004), la forma de realizar este cálculo es mediante la ecuación denominada Identidad de la Contabilidad Nacional la cual se define como:

$$
Y=C+I+G+X N \text {. }
$$

Donde:

$Y=\mathrm{PIB}$ (unidades monetarias).

$C=$ Consumo (gasto de familias).

$I=$ Inversión (gasto de las empresas).

$G=$ Compras del estado (gasto del gobierno).

$X N=$ Exportaciones neta $=$ exportacionesimportaciones.

El consumo se relaciona con los bienes y servicios que obtienen los hogares (Mankiw, 2006), la inversión se define

Ordaz, O., Marín, L., \& Gómez, D. 
como aumento de la cantidad física de capital Dornbusch et al., 2004), las compras de Estado es todo aquello que compran las administraciones públicas (Mankiw, 2006) y que también comprende los gastos destinados a la defensa nacional, la construcción de carreteras por parte de las administraciones locales y regionales y los sueldos de los funcionarios públicos (Dornbusch et al., 2004). Por último, las exportaciones netas es todo aquello que se exporta a los distintos países menos lo que se importa, además representan el gasto neto y proporciona la renta a los productores interiores (Mankiw, 2006).

De esta manera el PIB es un instrumento que se utiliza para medir la actividad económica de los países, con cálculos estadísticos y normas internacionales (Stiglitz et al., 2008), con esto se pueden realizar comparaciones entre países año tras año mediante los rendimientos que se obtengan (Krugman, Olney y Wells, 2008), además muestra la salud de la economía de cada país y es un parámetro para interpretar su crecimiento económico, aumentando así su productividad (Secretaría de Economía, 2017).

Según Heath (2012) el PIB es la medida que explica de mejor manera la actividad económica de México y que dentro de estas actividades económicas se encuentra el sector de

Medición de tiempo y frecuencia en las empresas 
telecomunicaciones el cual ha tenido influencia en el crecimiento económico del país. Además, de acuerdo con Bancomext (2015) las telecomunicaciones son un sector líder en la economía de México.

Sector de telecomunicaciones

El sector de telecomunicaciones (comunicar a distancia) ha tenido un alto crecimiento tanto a nivel nacional como internacional. Según el informe sectorial de telecomunicaciones en Chile del 2012 se muestra que tanto el PIB como el sector de comunicaciones crecieron muy a la par con el $5.66 \%$ el PIB de comunicaciones y $5.72 \%$ el PIB total (Subsecretaría de Telecomunicaciones de Chile, 2012). Mientras que en Argentina las telecomunicaciones son una industria con importantes externalidades positivas al desempeño económico (Montoya y Olarte, 2005) con impactos positivos del $0.15 \%$ por cada incremento del $1 \%$ en la penetración, confirmando así que las telecomunicaciones son un eje importante para la economía (Katz, Martin y Rentería, 2016).

En México la participación del sector de telecomunicaciones en el PIB es importante ya que ha existido un crecimiento continuo de este sector y para muestra de ello en

Ordaz, O., Marín, L., \& Gómez, D. 
el año 2004 existe un aumento del 4.4\% en el PIB, con un crecimiento equivalente del sector de las telecomunicaciones del $13.1 \%$ (Piedras, 2012). En este año el mayor crecimiento fue en los servicios por satélite, los cuales ofrecen señales de comunicación y los principales usuarios son las estaciones de radio y televisión (Instituto Nacional de Estadística y Geografía -INEGI-, 2004). Por esta razón las empresas de telecomunicaciones han integrado en sus procesos productivos equipos de cómputo cada vez más potentes, con la finalidad que este sector tenga más presencia en la economía nacional del país (INEGI ,2004).

Hasta ahora se ha analizado como las telecomunicaciones tienen un crecimiento continuo y por ende ocasionan que el PIB aumente, pero también es conveniente observar la fiabilidad y la alta capacidad de respuesta que se tienen ante la gran cantidad de información compartida e intercambiada entre los usuarios las redes de telecomunicaciones, para ello se abordara el tema de ingeniería de tráfico en las telecomunicaciones.

Medición de tiempo y frecuencia en las empresas 
Ingeniería de tráfico en las telecomunicaciones.

La necesidad de prepararse para el crecimiento en las líneas de comunicación en las diferentes áreas del país conlleva a la planeación, dimensión, desarrollo y la proyección de las redes de telecomunicaciones optimas (Carrión, 2012). El tráfico es en esencia "El tiempo de ocupación en los equipos de telecomunicaciones que tiene el usuario para lograr comunicarse" (Carrión, 2012).

La ingeniería de tráfico permite manipular el flujo de datos para que se ajuste a la red y no existan recursos que se utilicen innecesariamente que ocasione perdidas en el enlace (Argüello, 2015). La ingeniería de tráfico tiene dos parámetros esenciales; la razón de las llamadas (número de ocasiones que una trayectoria es usada por un periodo unitario) y el tiempo de espera que dura una llamada, con esto se puede decir que el tráfico se puede medir en tiempo en que se usa la línea y el número de veces en que existe comunicación y la duración de estas (Espinoza, 2008).

En general la ingeniería de tráfico se puede definir como el "proceso de distribuir sobre toda la topología de la red el

Ordaz, O., Marín, L., \& Gómez, D. 
tráfico circundante, con el fin de evitar congestiones y enlaces saturados" (López y Gelevez, p. 86, 2009).

Los objetivos que tiene la ingeniería de tráfico es maximizar los recursos existentes en las redes de telefonía, cambiar la ruta de la conexión electrónica en dado caso que esté muy congestionada para poder comunicarse y optimizar los recursos que se tienen para el control de tráfico (López y Gelevez, 2009).

La unidad que se utiliza para la medición del tráfico es el Erlang (Erl), el cual significa "cantidad de horas de ocupación en un grupo de órganos" (Carrión, 2012).

\section{Metodología}

La metodología de investigación del presente estudio busca determinar el impacto en el PIB del tráfico de información medido en tiempo y frecuencia de las distintas redes de telefonía en las empresas de telecomunicaciones. El tipo de metodología que se utiliza es cuantitativo, debido a que se hace un análisis econométrico. Con lo cual este trabajo puede resumirse en los siguientes pasos metodológicos:

Medición de tiempo y frecuencia en las empresas 
1. En primer lugar, se realiza un modelo de regresión con un Análisis de la Covarianza (ANCOVA) para determinar cómo ha sido la contribución al PIB del sector de telecomunicaciones en México, mediante:

a. Se analiza si los datos tienen una distribución normal mediante prueba Jarque - Bera.

b. Definir la ecuación (2) para la realización del modelo de regresión ANCOVA.

$$
\begin{gathered}
Y_{i}=B_{1}+B_{2} D_{2 i}+B_{3} D_{3 i}+\cdots+B_{n} D_{n i} \\
+B_{n+1} X_{i}+U_{i}
\end{gathered}
$$

Donde:

$Y_{i}=$ variable de respuesta.

$X_{i}=$ variable cuantitativa.

$D_{i i}=$ variable dummy .

2. En una segunda etapa, realizar un análisis del comportamiento de la inversión en infraestructura de las empresas de telecomunicaciones en los últimos años, mediante estadística descriptiva e inferencial.

3. En una última etapa, realizar un análisis del tráfico de la información (el cual se mide en tiempo y frecuencia) de

Ordaz, O., Marín, L., \& Gómez, D. 
distintas compañías de telecomunicaciones que existen en México mediante un análisis de regresión simple y la prueba de Chow para identificar posibles cambios estructurales, con el propósito de determinar cuáles son las que ofrecen un mejor servicio, además de identificar cómo han ido evolucionando con respecto a la transmisión de información.

Las variables que se utilizarán para llevar a cabo este estudio serán las mostradas en la tabla 1, siguiendo la definición del PIB de la ecuación (1). Los datos necesarios para llevar a cabo este estudio se toman del instituto federal de telecomunicaciones (IFT) del año 2000 al 2017 en forma trimestral.

\begin{tabular}{|c|c|c|c|}
\hline Consumo & Inversión & $\begin{array}{c}\text { Compras de } \\
\text { Estado }\end{array}$ & $\begin{array}{c}\text { Exportacione } \\
\text { s netas }\end{array}$ \\
\hline
\end{tabular}

Medición de tiempo y frecuencia en las empresas 


\begin{tabular}{|c|c|c|c|}
\hline $\begin{array}{l}\text { Vehículo de } \\
\text { motor y } \\
\text { piezas, } \\
\text { alimentos, } \\
\text { vivienda, } \\
\text { asistencia } \\
\text { sanitaria }\end{array}$ & $\begin{array}{l}\text { Estructuras } \\
\text { (no } \\
\text { residencias), } \\
\text { equipos y } \\
\text { programas } \\
\text { informáticos, } \\
\text { telecomunica } \\
\text { ciones, } \\
\text { residencial }\end{array}$ & $\begin{array}{c}\text { Defensa } \\
\text { nacional, } \\
\text { gastos de } \\
\text { escuelas } \\
\text { públicas, } \\
\text { investigación, } \\
\text { creación de } \\
\text { carreteras }\end{array}$ & $\begin{array}{c}\text { Exportacione } \\
\text { s, } \\
\text { importaciones } \\
\text { (automóviles, } \\
\text { teléfonos, } \\
\text { móviles, } \\
\text { servicios } \\
\text { financieros, } \\
\text { máquinas y } \\
\text { herramienta) } \\
\text { Exportacione }\end{array}$ \\
\hline
\end{tabular}

Fuente: Elaboración propia de la desagregación del PIB real Jones (2009).

\section{Resultados}

En la tabla 2 se muestra el incremento del PIB nacional y el incremento del PIB en el sector de telecomunicaciones.

Tabla 2. Comportamiento del PIB de México, PIB de telecomunicaciones y contribución de las telecomunicaciones en el PIB de México

\section{PIB de México \\ PIB de \\ Telecomunicaciones}

Ordaz, O., Marín, L., \& Gómez, D. 


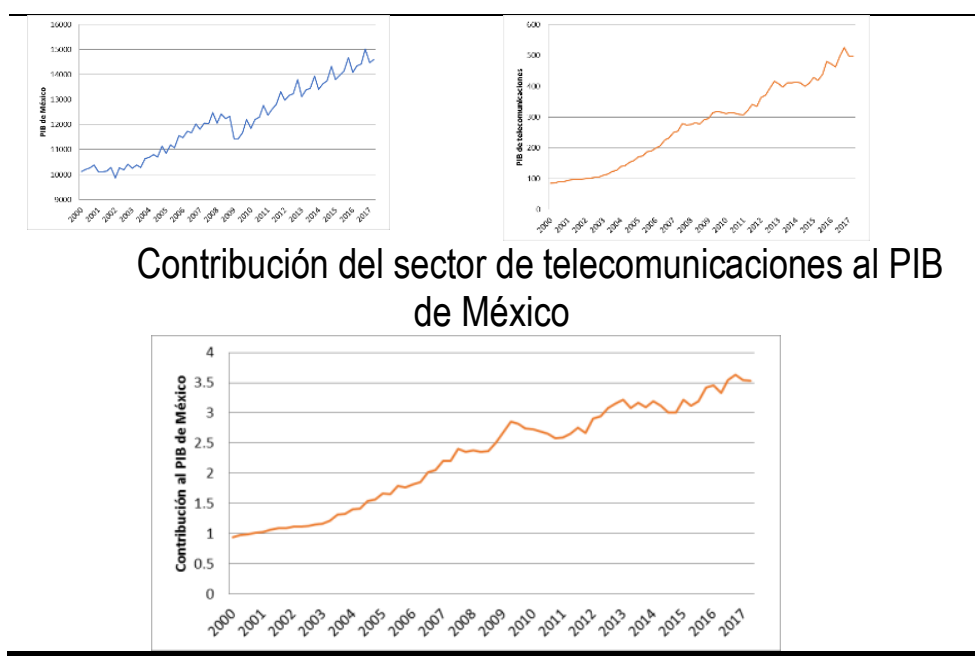

Fuente: Elaboración propia en base a los datos del IFT.

En la tabla 2 se observa como el PIB nacional ha ido creciendo a lo largo del tiempo, de igual manera lo ha hecho el PIB de telecomunicaciones, esto ocasiona que la contribución que existe sea cada vez mayor hasta llegar a porcentajes poco mayores al $3.5 \%$.

Para analizar con mayor profundidad el PIB Nacional y el PIB de telecomunicaciones primero se realizan pruebas de

Medición de tiempo y frecuencia en las empresas 
normalidad (Jarque- Bera) y posteriormente un modelo de regresión ANCOVA.

Tabla 3. Prueba jarque-bera PIB de telecomunicaciones

\section{Valores}

\begin{tabular}{lcc}
\multicolumn{1}{c}{ Pruebas } & No normalizados & Transformación no lineal \\
\hline Asimetría & -1.15 & -0.65 \\
Curtosis & 5.83 & 2.96 \\
Jarque Bera & 38.95 & 0.14 \\
Valor P & 0.00 & 0.93 \\
\hline
\end{tabular}

Fuente: Elaboración propia en base a datos del IFT.

En las pruebas de normalidad se observa primeramente que ninguna prueba acepta la normalidad de los datos con lo cual se procede a realizar una transformación lineal. Una vez hecho esto se encuentra que la curtosis es cercana a 3 con lo cual concluimos que los datos si se distribuyen normalmente, además el valor de la prueba Jarque Bera es cercano a cero y dado que el valor $\mathrm{P}$ es mayor que el valor de la prueba Jarque

Ordaz, O., Marín, L., \& Gómez, D. 
Bera, se puede aceptar que los datos se distribuyen normalmente.

Con esta transformación lineal se realiza un modelo de regresión ANCOVA en el cual se obtiene la ecuación (3):

$$
\begin{array}{cc}
\hat{y}_{i}=-0.033 & +0.069 X_{i}+0.049 D_{2}+0.032 D_{3}+ \\
0.064 D_{4} & \text { (3) }
\end{array}
$$

Dónde:

$\hat{y}_{i}=$ PIB promedio de México,

$X_{i}=\mathrm{PIB}$ de las telecomunicaciones,

$D_{2}=1$ si es el segundo trimestre del año; 0 en otro caso.

$D_{3}=1$ si es el tercer trimestre del año; 0 en otro caso.

$D_{4}=1$ si es el cuarto trimestre del año; 0 en otro caso.

\begin{tabular}{|c|c|c|c|c|c|c|}
\hline & $\begin{array}{l}\text { Interce } \\
\text { pto }\end{array}$ & $\begin{array}{c}\text { PIB de } \\
\text { telecomunicaciones }\end{array}$ & $\begin{array}{l}\text { segun } \\
\text { do }\end{array}$ & $\begin{array}{l}\text { imestre } \\
\text { terce } \\
\text { ro }\end{array}$ & $\begin{array}{l}\text { cuart } \\
0\end{array}$ & $R^{2}$ \\
\hline $\begin{array}{l}\text { Coeficien } \\
\text { tes }\end{array}$ & -0.033 & 0.069 & 0.049 & 0.032 & $\begin{array}{c}0.06 \\
4\end{array}$ & 0.77 \\
\hline $\begin{array}{l}\text { error } \\
\text { típico }\end{array}$ & 0.003 & 0.047 & 0.004 & 0.004 & $\begin{array}{c}0.00 \\
4\end{array}$ & 4 \\
\hline
\end{tabular}

Tabla 4. Valores del modelo de regresión ANCOVA

Medición de tiempo y frecuencia en las empresas 


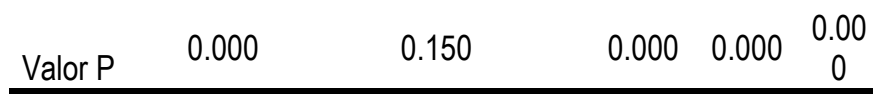

Fuente: Elaboración propia en base a la información del IFT.

El valor $R^{2}$ indica que el PIB de telecomunicaciones explica la variación del PIB nacional en un $77 \%$, de esta manera a un nivel de significancia del $5 \%$ se confirma que cada uno de los trimestres influye en el crecimiento del PIB. La interpretación de los resultados de la tabla 4 es la siguiente si el PIB de telecomunicaciones aumenta un $1 \%$, en promedio el PIB nacional se incrementa en $.069 \%$. También se puede afirmar que el cuarto trimestre del PIB de telecomunicaciones es el que más influye en el PIB nacional con $0.64 \%$.

Para poder obtener la contribución de las telecomunicaciones en el PIB se utiliza a la ecuación (3) un modelo log - log para encontrar la ecuación (4).

$$
\begin{gathered}
\ln \hat{y}_{i}=\ln (0.033)+\ln \left(0.069 X_{i}\right)+ \\
\ln \left(0.049 D_{2}\right)+\ln \left(0.032 D_{3}+\ln \left(0.064 D_{4}\right)\right. \\
\ln \hat{y}_{i}=3.41+\ln \left(0.069 X_{i}\right)+\ln \left(0.049 D_{2}\right) \\
+\ln \left(0.032 D_{3}\right)+\ln \left(0.064 D_{4}\right)
\end{gathered}
$$

Ordaz, O., Marín, L., \& Gómez, D. 
La gráfica 2 muestra que en los últimos cuatro años las empresas que más invierten en infraestructura son las redes de telefonía de Telefonos de México (TELMEX), TELCEL, TELEFONICA Y CABLEMAS, actualmente existen nuevas compañías que están entrando al sector de telecomunicaciones y no tienen gran impacto debido en gran medida a la absorción de diferentes compañías tal y como se muestra en la gráfica 3 , la cual muestra nuevamente como TELMEX, TELCEL, TELEFONICA y CABLEMAS, son las que tienen mayor inversión en su infraestructura, añadiéndose a estas la empresa AT\&T. De esta manera y a excepción de CABLEMAS, las redes de telefonía son las que mayor inversión en promedio realizan en su infraestructura.

Para tener un panorama de cómo ha sido el tráfico de información de las cuatro redes de telefonía que más han invertido en los últimos años en infraestructura, se muestra la gráfica 3 , en la cual se puede apreciar como el tráfico de información de la red de telefonía TELCEL es mucho mayor a las de las otras redes de telefonía, mientras que la red de telefonía TELEFONICA muestra un decrecimiento en su tráfico de información.

Medición de tiempo y frecuencia en las empresas 
Para cada una de las redes de telefonía con respecto al tráfico de información se realizó una prueba de Chow para determinar la existencia de un posible cambio estructural y un análisis de regresión simple para determinar el comportamiento a lo largo del tiempo.

Gráfico 1. Inversión promedio de la infraestructura de las empresas de telecomunicaciones

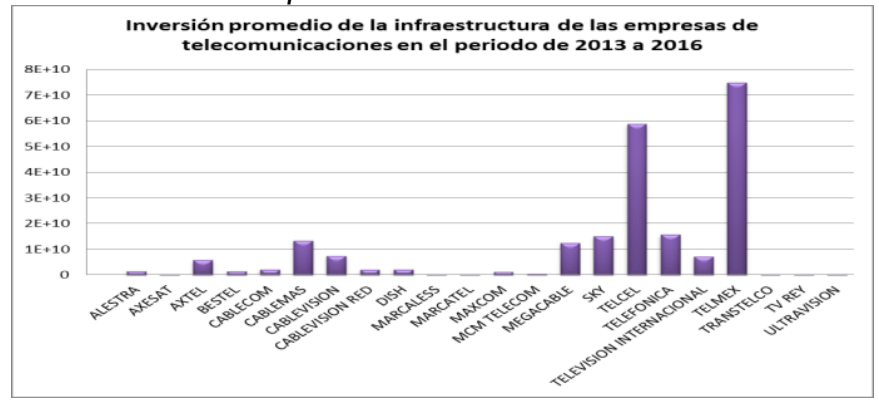

Fuente: Elaboración propia en Base a la información del IFT en un periodo de 2013-2016.

Ordaz, O., Marín, L., \& Gómez, D. 
Gráfico 2. Inversión promedio de nuevas y anteriores empresas

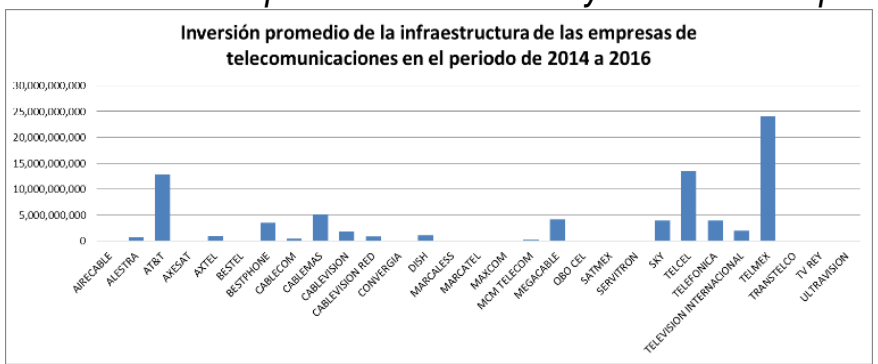

Fuente: Elaboración propia en base a la información obtenida en el IFT del periodo de 2014 a 2016.

Gráfico 3. Tráfico de información de las redes de telefonía

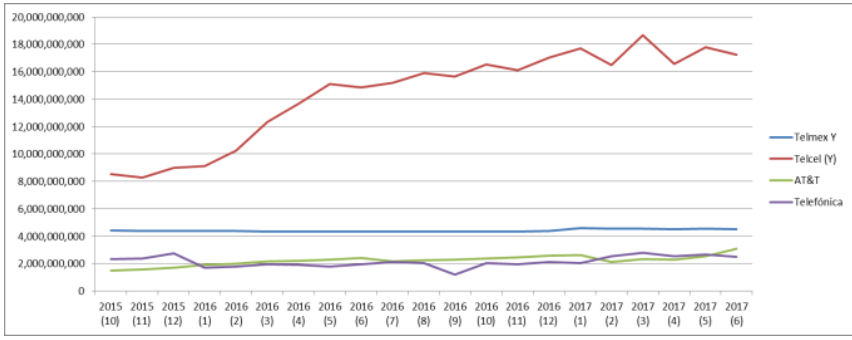

Fuente: Elaboración propia en base a la información obtenida en el IFT.

\section{Medición de tiempo y frecuencia en las empresas}


Tabla 5. Análisis de un cambio estructural en el tráfico de información en redes de telefonía

\begin{tabular}{lccccc}
\hline \multicolumn{7}{c}{ Cambio estructural } \\
\hline & Telcel & Telmex & AT\&T1 & AT\&T2 & Movistar \\
F Calculada & 21.894 & 24.025 & 5.016 & 3.45 & 9.291 \\
F crítica & 3.592 & 3.592 & 3.592 & 3.592 & 3.592 \\
P valor & 0.0001 & 0.0001 & 0.019 & 0.055 & 0.002 \\
\hline
\end{tabular}

Fuente: Elaboración propia en base a la información del IFT.

La tabla 5 muestra los valores de la prueba de Chow para cada una de las redes de telefonía. Al determinar los periodos de posible cambio estructural, en todas las telefonías se encontró un posible periodo de cambio estructural excepto para AT\&T, el cual mostro dos posibles cambios estructurales. Los resultados que se obtuvieron fueron que a un nivel de significancia del $5 \%$ existió un cambio de estructura debido al valor $P$. Por lo tanto, se puede analizar el tráfico de información con dos regresiones lineales para Telcel, Telmex y Movistar; $y$ en el caso de AT\&T con cuatro regresiones lineales.

La tabla 6 muestra los diferentes análisis de regresión tomando como variable independiente el tiempo y variable dependiente el tráfico de información.

Ordaz, O., Marín, L., \& Gómez, D. 
Tabla 6. Análisis de regresión del tráfico de información de

\begin{tabular}{|c|c|c|}
\hline \multicolumn{3}{|c|}{ Telcel } \\
\hline \multicolumn{3}{|c|}{ Telcel } \\
\hline & Octubre 2015 - Mayo 2016 & Junio 2016 - Junio 2017 \\
\hline Regresi & $Y_{t}=6269557769.39+100301$ & $Y_{t}=13234744137.86+223832$ \\
\hline & $4652.52+X_{t}$ & $584.837+X_{t}$ \\
\hline $\begin{array}{l}\text { Coeficieie } \\
\text { nte de } \\
\text { correlac }\end{array}$ & & \\
\hline ión & 0.946 & 0.798 \\
\hline$R^{2}$ & 0.878 & 0.604 \\
\hline Valor $\mathrm{P}$ & 0.000 & 0.001 \\
\hline
\end{tabular}

Fuente: Elaboración propia en base a la información del IFT.

En base al valor $\mathrm{P}$ y a un nivel de significancia del $5 \%$ se puede decir que el tiempo si influye en el comportamiento del tráfico de información, además el $R^{2}$ muestra que el tiempo explica la variación del tráfico de información en un $88 \%$ para el periodo de octubre 2015 a mayo 2016 el promedio del tráfico de información fue de 1,003 millones mientras en que en el otro periodo de junio de 2016 a junio de 2017 el tráfico de información es de 223.8 millones. De esta manera el tráfico de información de la primera regresión lineal es mayor a la segunda con lo que

Medición de tiempo y frecuencia en las empresas 
se puede discernir que éste se ha reducido al pasar los meses, pero aún existe un crecimiento en el tráfico de información.

Tabla 7. Análisis de regresión del tráfico de información de

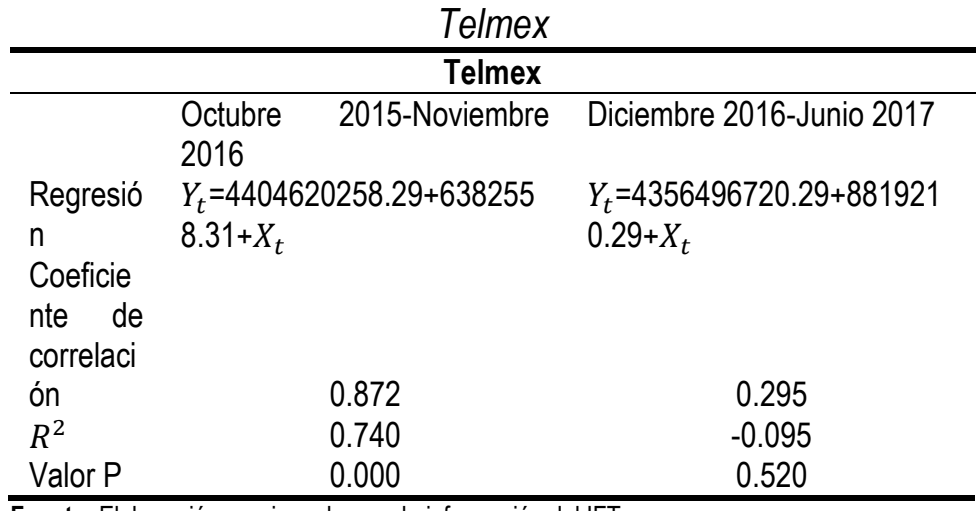

Fuente: Elaboración propia en base a la información del IFT.

La tabla 7 muestra que en un periodo de octubre 2015 a noviembre 2016, la variable tiempo si influye de manera significativa en el tráfico de información y con el $R^{2}$ se puede observar que el tiempo explica en un $74 \%$ y el comportamiento del tráfico de información respectivamente. Por otro lado, en el periodo de diciembre 2016 a junio 2017 la variable tiempo explica en un $9 \%$ el comportamiento del tráfico de información

Ordaz, O., Marín, L., \& Gómez, D. 
lo cual nos dice que esta variable está influenciada por otras variables. La regresión del primer periodo muestra que en promedio el tráfico de información es de 6.3 millones mucho menor al tráfico de información que existe entre con la compañía TELCEL.

Tabla 8. Análisis de regresión del tráfico de información de

\begin{tabular}{|c|c|c|}
\hline \multicolumn{3}{|c|}{$A T \& T$} \\
\hline AT\&T & Octubre 2015 - Junio 2016 & Julio 2016 - Junio 2017 \\
\hline & $\begin{array}{l}Y_{t} \\
=1373713837.72\end{array}$ & $\begin{array}{l}Y_{t} \\
=1773553584.69\end{array}$ \\
\hline $\begin{array}{l}\text { Regresión } \\
\text { Coeficiente de }\end{array}$ & $+118429265.23 X_{t}$ & $+41737417.28 X_{t}$ \\
\hline correlación & 0.990 & 0.570 \\
\hline$R^{2}$ & 0.977 & $\begin{array}{l}0.258 \\
0.053\end{array}$ \\
\hline Valor $\mathrm{P}$ & 0.000 & \\
\hline AT\&T & $\begin{array}{l}\text { Octubre } 2015 \text { - Febrero } \\
2017\end{array}$ & Marzo 2017 - Junio 2017 \\
\hline & $\begin{array}{l}Y_{t} \\
=1657377158.76\end{array}$ & $\begin{array}{l}Y_{t} \\
=250884218.3 \mathrm{X}\end{array}$ \\
\hline $\begin{array}{l}\text { Regresión } \\
\text { Coeficiente de } \\
\text { correlación }\end{array}$ & $\begin{array}{c}+54289823.59 X_{t} \\
0.835\end{array}$ & $\begin{array}{c}-2328044907.6 X_{t} \\
0.892\end{array}$ \\
\hline
\end{tabular}

Medición de tiempo y frecuencia en las empresas 
$\begin{array}{lll}R^{2} & 0.677 & 0.693 \\ \text { Valor P } & 0.000 & 0.108\end{array}$

Fuente: Elaboración propia en base a la información del IFT.

En la red de telefonía AT\&T se analizaron dos periodos en los que se obtuvieron dos cambios estructurales, en los tres primeros periodos la variable tiempo si es significativa dado un nivel de confianza de $10 \%$, el último periodo no es significativo bajo este mismo nivel de significancia debido en gran medida a que el número de datos con los cuales se realiza la regresión son muy pocos. De esta manera y como se realizó en la tabla anterior no se analizará el último periodo. En el segundo periodo con el $R^{2}$ de 0.258 se observa que la variable tiempo no explica el comportamiento del tráfico de información, con lo cual se analizó que es lo que sucede el en el primer y tercer periodo. En el primer periodo el tráfico de información en promedio es 118.4 millones mientras que el tercer periodo el tráfico de información es de 54.2 millones lo que al analizar un periodo más grande se observa que el crecimiento es mayor en promedio del tráfico de información.

Ordaz, O., Marín, L., \& Gómez, D. 
Tabla 9. Análisis de regresión del tráfico de información de movistar.

\begin{tabular}{lcc}
\hline \multicolumn{1}{c}{ Movistar } & Octubre 2015 - Septiembre & Octubre 2016 - Junio \\
\hline & 2016 & 2017 \\
Regresión & $Y_{t}=2410752062.62$ & $Y_{t}=793325337.07$ \\
Coeficiente de & $+65023007.61+X_{t}$ & $+91704246.8+X_{t}$ \\
correlación & & \\
$R^{2}$ & 0.601 & 0.806 \\
Valor P & 0.298 & 0.599 \\
\hline
\end{tabular}

Fuente: Elaboración propia en base a la información del IFT.

En la tabla 9 se observa como la variable tiempo si es significativa dado un nivel de confianza del $5 \%$ con lo que se puede, aunque para el primer periodo el tiempo no explica el tráfico de información muy bien dado que el $R^{2}$ es de 0.298 lo que nos dice que existen otras variables que influyen en el tráfico de información, en el segundo periodo también se muestra que el $R^{2}$ es muy bajo, aun así se observa que en promedio el tráfico de información es de 91.7 millones mucho mayor al tráfico de información de AT\&T y TELMEX.

Medición de tiempo y frecuencia en las empresas 


\section{Conclusiones}

Este trabajo tiene una investigación del tipo cuantitativo que muestra la contribución y el impacto que tiene el PIB de telecomunicaciones en el PIB de México en un periodo de 18 de años, también se muestra como ha sido la inversión en infraestructura en el sector de telecomunicaciones y como ha sido el tráfico de información de las redes de telefonía en los últimos 3 años.

Los resultados muestran que la contribución del PIB de telecomunicaciones en el PIB nacional ha aumentado a más del $3.5 \%$, además en el cuarto cuatrimestre del año es donde se muestra una mayor contribución al PIB de México.

Con respecto a la inversión en infraestructura en el sector de telecomunicaciones, muestra que TELCEL. TELMEX, TELEFONICA son las que mayor aporte han tenido en los últimos años, además con la reforma de telecomunicaciones del 2014 permitió a la compañía de AT\&T entrar en el mercado mexicano y hacer fuertes inversiones con la compra de redes de telefonía como NEXTEL y IUSACELL.

La empresa TELMEX es la que mayor inversión ha tenido en los últimos años, posteriormente le sigue TELCEL, y entre

Ordaz, O., Marín, L., \& Gómez, D. 
estas dos la que tienen mayor tráfico de información con respecto al número de llamadas que realizan es la empresa TELCEL, con lo cual es la que acapara mayor parte en el mercado.

\section{Referencias}

Banco Nacional de Comercio Exterior (2015). Sectorial: telecomunicaciones (1er informe). Recuperado de: http://www.bancomext.com/wpcontent/uploads/2015/08/Telecomunicaciones_1er_Informe_2015.p df

Cayuela, L. (2010). Modelos lineales: regresión, ANOVA y ANCOVA. Disponible en: https://portal.uah.es/portal/page/portal/epd2_asignaturas/asig2022 18/informacion_academica/2-Modelos\%20lineales.pdf

Dornbusch, R., Fisher, S., Startz, R. (2004). Macroeconomía. Colombia: McGraw-Hill, Inc.

Fernández, G. (2005) Econometría. Madrid, España: Pearson educación, S.A.

Galindo, P. (2015). El crecimiento económico no implica crecer en bienestar 6 indicadores alternativos al Producto Interno Bruto. El salmón contracorriente. Disponible en: http://www.elsalmoncontracorriente.es/?6-indicadores-alternativosal

\section{Medición de tiempo y frecuencia en las empresas}


Garcia V (1991) Predicción en modelos no lineales. Disponible en: http://renanquispellanos.com/recursos/Aporte\%20Intelectual/Tecnic as\%20Prediccion/10.unidad7.pdf

García, P. (2007). La evolución de las telecomunicaciones. EGAP Working Papers 2007-02. Disponible en: http://alejandria.ccm.itesm.mx/egap/documentos/EGAP-200702.pdf

Hardy, A. P. (1980). The role of the telephone in economic development. Telecommunications Policy, 4(4), 278-286. doi: 10.1016/03085961(80)90044-0

Heath, J. (2012). Lo que indican los indicadores. Instituto Nacional de Estadística y Geográfia, Mexico, (10mo informe). Disponible en: http://www.academia.edu/download/45283243/Lo_que_indican_los indicadores.pdf

Instituto Nacional de Estadística y Geografía (1999). Actividades informáticas y de telecomunicaciones. Disponible en: http://www3.inegi.org.mx/sistemas/componentes/previsualizador/vi sta.aspx?arch=/prod_serv/contenidos/espanol/bvinegi/productos/ce nsos/economicos/1999/general/telecomuni.pdf\&tipo=1

Instituto Nacional de Estadística y Geografía (2004). Actividades relacionadas con la informática y las telecomunicaciones en México. Disponible en: https://www.inegi.org.mx/app/biblioteca/ficha.html?upc=702825001 370

Jones, C. (2009). Macroeconomía. Barcelona, España: Antoni Bosch editor.

Ordaz, O., Marín, L., \& Gómez, D. 
Katz, R., Martin, F., Rentería, C. (2016). Evaluación del impacto económico de las telecomunicaciones en Argentina. CPRLATAM Conference. Disponible en: https://ssrn.com/abstract $=2877570$

Krugman, P., Olney, M., \& Wells, Rubens (3era ed.). (2008). Fundamentos de economía. Barcelona, España: Reverté

Kuznets, S. (1934). National Income, 1929-1932. National Bureau of Economic Research. Disponible en: https://www.nber.org/chapters/c2258.pdf

Madrid-Aris, M. (s.f.). Terminos técnicos de telecomunicaciones. Public Utility Research Center. Disponible en: http://bear.warrington.ufl.edu/centers/purc/DOCS/papers/sp_02.pdf

Mankiw, G. (2006). Macroeconomía. Barcelona, España: Antoni Bosch.

Mankiw, N. G. (2006). The Macroeconomist as Scientist and Engineer. Journal of Economic Perspectives, 20(4), 29-46. doi:10.1257/jep.20.4.29

Montoya, A., y Olarte, A. (2005). El TLC y el sector de las telecomunicaciones en Colombia: panorama actual. Perfil de coyuntura económica. 7293. https://aprendeenlinea.udea.edu.co/revistas/index.php/coyuntura/ar ticle/download/2302/1861

Moreno, I. (2003). Protocolo RTP y Protocolo SIP. Redes de computadora. Universidad de Sonora. México.

My Structured Query Language hispano (2003). Normalización de bases de datos.

en: http://www.eet2mdp.edu.ar/alumnos/MATERIAL/MATERIAL/info/inf onorma.pdf

\section{Medición de tiempo y frecuencia en las empresas}


Pérez, S., Blanca, R., García, M. (2010). Análisis del cambio estructural en el modelo de regresión lineal. Revista de Matemática: Teoría y Aplicaciones, $\quad 17(2)$ Disponible en: https://www.redalyc.org/articulo.oa?id=453/45326933004

Piedras, E. (2012). Regulación y desempeño del sector de telecomunicaciones: el impacto en el bienestar, la productividad y la competitividad de México. Centro de investigación y docencia Económicas. Disponible en: http://nszjmka.razonypalabra.org.mx/anteriores/n49/bienal/Mesa\%2 012/ErnestoPiedras.pdf

Sánchez, P. (2008). Cambios estructurales en series de tiempo: una revisión del estado del arte. Revista Ingenierías, 7(12). Disponible en: https://revistas.udem.edu.co/index.php/ingenierias/article/view/202/ 189

Secretaría de Economía (2017). ¿Para qué sirve el PIB?. México. Disponible en: $\quad$ http://www.2006-2012.economia.gob.mx/economia-paratodos/tema-del-dia/6950-para-que-sirve-el-pib

Stephen, J. (2005). Econometría. México: McGraw-Hill Companies, Inc.

Stiglitz, J., Sen, A. y Fitoussi, J.P. (2008). Informe de la comisión sobre medición del desarrollo económico y del progreso social. Comisión Stiglitz. Disponible en: http://www.palermo.edu/Archivos_content/2015/derecho/pobreza multidimensional/bibliografia/Biblio_adic5.pdf

Subsecretaría de Telecomunicaciones (2012). Informe Sectorial: telecomunicaciones en chile. Disponible en: https://www.subtel.gob.cl/images/stories/apoyo_articulos/notas_pre

Ordaz, O., Marín, L., \& Gómez, D. 
nsa/cta_publica_2010_2014/capitulo_telecomunicaciones_060120 14.pdf

Zubcoff, J. (2010). ANCOVA. Universidad de Alicante. Disponible en: https://rua.ua.es/dspace/bitstream/10045/4171/1/DE\%20\%204\%20-\%20ANCOVA.pdf

Medición de tiempo y frecuencia en las empresas 